\title{
Intrusion errors moderate the relationship between blood glucose and regional cerebral blood flow in cognitively unimpaired older adults
}

\author{
Kelsey R. Thomas ${ }^{1,2}$ (1) - Alexandra J. Weigand ${ }^{3} \cdot$ Isabel H. Cota ${ }^{1}$. Emily C. Edmonds ${ }^{1,2} \cdot$ Christina E. Wierenga $^{1,2} \cdot$ \\ Mark W. Bondi ${ }^{2,4} \cdot$ Katherine J. Bangen ${ }^{1,2} \cdot$ for the Alzheimer's Disease Neuroimaging Initiative
}

Accepted: 29 June 2021 / Published online: 20 August 2021

This is a U.S. government work and not under copyright protection in the U.S.; foreign copyright protection may apply 2021

\begin{abstract}
Regional cerebral blood flow (CBF) has a complex relationship with cognitive functioning such that cognitively unimpaired individuals at risk for Alzheimer's disease (AD) may show regional hyperperfusion, while those with cognitive impairment typically show hypoperfusion. Diabetes and word-list intrusion errors are both linked to greater risk of cognitive decline and dementia. Our study examined associations between fasting blood glucose, word-list intrusion errors, and regional CBF. 113 cognitively unimpaired older adults had arterial spin labeling MRI to measure CBF in a priori AD vulnerable regions: medial temporal lobe (MTL), inferior parietal lobe (IPL), precuneus, medial orbitofrontal cortex (mOFC), and pericalcarine (control region). Hierarchical linear regressions, adjusting for demographics, vascular risk, and reference CBF region, examined the main effect of blood glucose on regional $\mathrm{CBF}$ as well as whether intrusions moderated this relationship. Higher glucose was associated with higher $\mathrm{CBF}$ in the precuneus $(\beta=.134,95 \% \mathrm{CI}=.007$ to $.261, p=.039)$, IPL $(\beta=.173,95 \% \mathrm{CI}=.072$ to $.276, p=.001)$, and mOFC ( $\beta=.182,95 \% \mathrm{CI}=.047$ to $.320, p=.009)$. There was no main effect of intrusions on CBF across regions. However, the glucose $\mathrm{x}$ intrusions interaction was significant such that having higher glucose levels and more intrusion errors was associated with reduced CBF in the MTL ( $\beta=-.186,95 \% \mathrm{CI}=-.334$ to $-.040, p=.013)$ and precuneus $(\beta=-.146,95 \% \mathrm{CI}=-.273$ to $-.022, p=.022)$. These findings may reflect early neurovascular dysregulation, whereby higher $\mathrm{CBF}$ is needed to maintain unimpaired cognition in individuals with higher glucose levels. However, lower regional CBF in unimpaired participants with both higher glucose and more intrusions suggests a failure in this early compensatory mechanism that may signal a decrease in neural activity in $\mathrm{AD}$ vulnerable regions.
\end{abstract}

Keywords Cerebral blood flow · Glucose $\cdot$ Neuropsychology $\cdot$ Process scores $\cdot$ Intrusion errors · Alzheimer's disease

\section{Introduction}

Data used in preparation of this article were obtained from the Alzheimer's Disease Neuroimaging Initiative (ADNI) database (adni.loni.usc.edu). As such, the investigators within the ADNI contributed to the design and implementation of ADNI and/or provided data but did not participate in analysis or writing of this report. A complete listing of ADNI investigators can be found at: http://adni.loni.usc.edu/wp-content/uploads/how_to_apply/ADNI_ Acknowledgement_List.pdf.

Kelsey R. Thomas

kthomas@health.ucsd.edu

1 Research Service, VA San Diego Healthcare System, 3350 La Jolla Village Drive (151), San Diego, CA, USA

2 Department of Psychiatry, University of California, San Diego, La Jolla, CA, USA
The co-occurrence of cerebrovascular and Alzheimer's disease (AD)-related pathologies are extremely common (Kapasi et al., 2017). Growing evidence suggests that cerebrovascular changes may either accelerate AD-related cognitive decline or be a defining feature of $\mathrm{AD}$ (Bangen et al., 2015, 2017; Lee et al., 2016; Zlokovic, 2011). One indicator

3 San Diego State University/University of California, San Diego Joint Doctoral Program in Clinical Psychology, San Diego, CA, USA

4 Psychology Service, VA San Diego Healthcare System, San Diego, CA, USA 
of cerebrovascular function is cerebral blood flow (CBF), which has consistently shown a complex relationship with cognitive functioning across the $\mathrm{AD}$ continuum such that cognitively unimpaired (CU) individuals at risk for $\mathrm{AD}$ may show regional hyperperfusion, while those with frank cognitive impairment show hypoperfusion (Bangen et al., 2012; Fleisher et al., 2009; Mattsson et al., 2014; Thomas et al., 2021; Wierenga et al., 2014).

Type 2 diabetes mellitus (T2DM) is a vascular/metabolic disease that is a risk factor for mild cognitive impairment (MCI) and dementia, including AD dementia (Livingston et al., 2020; Luchsinger et al., 2007). Prior work has shown that T2DM is associated with reduced CBF (Bangen et al., 2018) and also modifies the relationship between AD risk factors (e.g., apolipoprotein E [APOE] $\varepsilon 4$ allele, subtle cognitive decline, tau), and future functional decline (Thomas, et al., 2020a, b). Given that even pre-diabetes is associated with higher risk of cognitive impairment (Yaffe et al., 2004), the current study examined the relationship between fasting blood glucose (FBG), which is higher among individuals with pre-diabetes and diabetes, and regional CBF.

Several studies have shown that subtle cognitive inefficiencies, measured using neuropsychological process and error scores, can be captured in the preclinical phase of AD (Abulafia et al., 2018; Loewenstein et al., 2018) and that these sensitive scores add prognostic value to predict future progression to $\mathrm{MCI} / \mathrm{dementia}$, above and beyond traditional AD biomarkers (Thomas et al., 2018). Intrusion errors, defined as non-target words said during a word-list memory test, have been shown to be particularly sensitive to early AD-related changes (Bondi et al., 1999; Thomas et al., 2018). Intrusion errors are not considered in calculating the total learning, recall, and recognition scores on traditional word-list measures, and are often overlooked in cohort studies of aging and AD. Recent work has demonstrated that participants with subtle cognitive difficulties, including those with impaired process scores such as intrusion errors, had higher hippocampal CBF than CU or MCI participants (Thomas et al., 2021), such that an inverted-U pattern of regional CBF changes was observed across diagnostic stages.

Regional CBF appears to be an early biomarker of risk for AD-related declines and has been previously associated with both T2DM and subtle cognitive difficulties. In turn, both T2DM and intrusion errors have been shown to confer greater risk of cognitive decline and progression to $\mathrm{MCI} /$ dementia. Thus, the current study aimed to examine the associations between FBG, intrusion errors, and CBF in regions vulnerable to early $\mathrm{AD}$ pathology. Furthermore, the moderating effect of intrusion errors on the associations between FBG and regional CBF was examined. We hypothesized that having both higher FBG and more intrusion errors would be associated with lower regional CBF due to a failure in expected neural compensation.

\section{Methods}

Data used in the preparation of this article were obtained from the Alzheimer's Disease Neuroimaging Initiative (ADNI) database (adni.loni.usc.edu). The ADNI was launched in 2003 as a public-private partnership, led by Principal Investigator Michael W. Weiner, MD. The primary goal of ADNI has been to test whether serial magnetic resonance imaging (MRI), positron emission tomography (PET), other biological markers, and clinical and neuropsychological assessment can be combined to measure the progression of mild cognitive impairment (MCI) and early Alzheimer's disease (AD). For up-to-date information, see www.adniinfo.org.

\section{Participants}

Detailed inclusion criteria for ADNI have been reported elsewhere (Petersen et al., 2010). Briefly, at enrollment, participants were aged 55-90 years, had $>5$ years of education or work-history equivalent, had a Geriatric Depression Scale <6, were fluent in English or Spanish, and were generally healthy (Hachinski score $<5$ ). For this study, all participants denied a clinical history of stroke and were considered cognitively unimpaired (CU), which was defined as not having dementia based on the baseline ADNI assessment (McKhann et al., 1984; Petersen et al., 2010) as well as not being classified as having MCI based on Jak/Bondi actuarial neuropsychological criteria (Bondi et al., 2014; Jak et al., 2009; see Supplemental Methods for description of criteria).

The current study included $113 \mathrm{CU}$ participants from ADNI GO/ADNI 2 cohorts who had arterial spin labeling (ASL) MRI data within 12 months of their baseline visit, $\left[{ }^{18} \mathrm{~F}\right]$-fluorodeoxyglucose (FDG)-PET imaging, and FBG data available. Sample characteristics are shown in Table 1.

\section{Structural and arterial spin labeling MRI}

Detailed information describing MRI data acquisition, processing, and analysis is available online at http://adni.loni. usc.edu/. Briefly, the T1-weighted 3D MPRAGE structural MRI and ASL scan were done at the same visit and performed on a 3.0 Tesla scanner (Siemens).

Pulsed ASL scans were acquired using QUIPS II with thin-slice T11 periodic saturation sequence ("Q2TIPS") with echo-planar imaging (Luh et al., 1999). The sequence incorporated the following set parameters: inversion time of arterial spins $(\mathrm{T} 11)=700 \mathrm{~ms}$, total transit time of the spins $(\mathrm{T} 12)=1900 \mathrm{~ms}$, tag thickness $=100 \mathrm{~mm}$, tag to 
Table 1 Sample $(N=113)$ demographic and descriptive characteristics

\begin{tabular}{|c|c|c|c|}
\hline & Mean or $\%$ & $\mathrm{SD}$ or $\mathrm{N}$ & Range \\
\hline Age, years & 70.75 & 6.39 & $59.50-84.70$ \\
\hline Education, years & 16.58 & 2.48 & $12-20$ \\
\hline Female & $53.1 \%$ & $N=60$ & - \\
\hline White & $94.7 \%$ & $N=107$ & - \\
\hline Hispanic/Latino & $4.4 \%$ & $N=5$ & - \\
\hline APOE $\varepsilon 4$ carrier & $33.6 \%$ & $N=38$ & - \\
\hline MMSE & 29.04 & 1.08 & $25-30$ \\
\hline FDG-PET SUVR & 1.30 & 0.12 & $0.98-1.66$ \\
\hline CSF $p$-tau/A $\beta$ positive* & $26.8 \%$ & $N=26$ & - \\
\hline BMI & 27.00 & 4.57 & $18.71-51.35$ \\
\hline Systolic blood pressure & 135.22 & 16.72 & $101-195$ \\
\hline Diastolic blood pressure & 74.54 & 9.84 & $50-102$ \\
\hline Pulse pressure & 60.68 & 15.18 & 34-109 \\
\hline $\mathrm{FBG}, \mathrm{mg} / \mathrm{dL}$ & 98.90 & 16.71 & $55-154$ \\
\hline Diabetes & $12.4 \%$ & $N=14$ & - \\
\hline $\begin{array}{l}\text { On hypoglycemic medica- } \\
\text { tion }\end{array}$ & $5.3 \%$ & $N=6$ & - \\
\hline $\begin{array}{l}\text { Prediabetes } \\
\qquad(\mathrm{FBG}>100 \mathrm{mg} / \mathrm{dL})\end{array}$ & $33.6 \%$ & $N=38$ & - \\
\hline Total intrusion errors & 4.00 & 4.40 & $0-24$ \\
\hline \multicolumn{4}{|l|}{ CBF by ROI (mL/100 g/min) } \\
\hline MTL & 26.54 & 7.00 & $8.11-46.51$ \\
\hline Precuneus & 31.29 & 10.27 & $8.49-57.46$ \\
\hline Inferior Parietal Lobe & 31.84 & 9.83 & $12.14-67.77$ \\
\hline $\begin{array}{l}\text { Medial Orbital Frontal } \\
\text { Cortex }\end{array}$ & 23.92 & 7.34 & $7.40-47.71$ \\
\hline Pericalcarine & 42.93 & 11.96 & $16.49-76.70$ \\
\hline MTL volume $\left(\mathrm{mm}^{3}\right)$ & 5645.14 & 705.03 & $3766.00-7027.00$ \\
\hline \multicolumn{4}{|l|}{ Cortical Thickness (mm) } \\
\hline Precuneus & 2.31 & 0.12 & $1.91-2.65$ \\
\hline Inferior Parietal Lobe & 2.41 & 0.14 & $1.96-2.68$ \\
\hline $\begin{array}{l}\text { Medial Orbital Frontal } \\
\text { Cortex }\end{array}$ & 2.32 & 0.12 & $2.04-2.71$ \\
\hline Pericalcarine & 1.55 & 0.11 & $1.34-1.93$ \\
\hline
\end{tabular}

$A P O E=$ apolipoprotein E; $M M S E=$ Mini Mental State Exam; $F D G-P E T=[18 \mathrm{~F}]$ fluorodeoxyglucose positron emission tomography; $C S F=$ cerebrospinal fluid; $\mathrm{p}$-tau $/ \mathrm{A} \beta=$ phosphorylated $\operatorname{tau} / \beta$ amyloid ratio; $B M I=$ body mass index; $F B G=$ fasting blood glucose; $M T L=$ medial temporal lobe. All participants on hypoglycemic medications were taking Metformin; there were no participants on insulin *available on subsample only $(n=97)$

proximal slice gap $=25.4 \mathrm{~mm}$, repetition time $=3400 \mathrm{~ms}$, echo time $=12 \mathrm{~ms}$, field of view $=256 \mathrm{~mm}, 64 \times 64$ matrix, 24 4-mm thick axial slices [52 tag + control image pairs], time lag between slices $22.5 \mathrm{~ms}$. Additional ASL data collection and processing methods used to derive CBF maps are in Supplemental Methods.

FreeSurfer (version 5.1; http://surfer.nmr.mgh.harvard. edu/fswiki) was used to skull-strip, segment, and parcellate the structural scans. FreeSurfer-derived anatomical regions of interest (ROIs) were applied and used to extract mean $\mathrm{CBF}$ and volume/cortical thickness for each participant. Mean CBF corrected for partial volume effects was extracted for each ROI and reference region for each hemisphere separately and any hemispheric differences in the main outcomes are reported in the results. To reduce the number of comparisons, however, averaged bilateral estimates for each ROI were used as the outcome variable in our models. We examined the following five a priori ROIs: (1) medial temporal lobe (MTL), which included the hippocampus and entorhinal cortex, (2) precuneus, (3) inferior parietal lobe (IPL), (4) medial orbitofrontal cortex (mOFC), and (5) pericalcarine cortex. The first four ROIs were chosen given prior work showing these regions are vulnerable to early AD-related changes and have associations between $\mathrm{CBF}$ and $\mathrm{AD}$ severity (Bangen et al., 2017; Dickerson et al., 2011; Mattsson et al., 2014; Thomas et al., 2021; Yew \& Nation, 2017). The pericalcarine ROI was selected as a control region to examine specificity of any results given that there are no expected changes in AD (Mattsson et al., 2014; Sanchez et al., 2020). Consistent with other CBF work in ADNI (Mattsson et al., 2014; Thomas et al., 2021; Yew \& Nation, 2017), CBF of the precentral gyrus was selected to serve as a covariate in the models, as it is not thought to be impacted in early $\mathrm{AD}$, allowing for adjustment of expected individual differences in $\mathrm{CBF}$ that are likely not due to $\mathrm{AD}$ pathologies. If participants were missing baseline ASL data but had ASL data within the first year of their baseline visit, the first occasion of ASL data was used in our analyses.

\section{Cerebral metabolism}

FDG scanning began 30-60 min after intravenous injection of an approximately $5 \mathrm{mCi}$ dose of tracer. PET images were spatially normalized to a Montreal Neurological Institute (MNI) PET template. In line with prior FDG-PET studies (Jagust et al., 2010; Landau et al., 2011), a composite metaROI was comprised of standardized uptake value ratios (SUVRs) from the left and right angular gyri, left and right middle/inferior temporal gyri, and bilateral posterior cingulate gyrus. The meta-ROI was intensity normalized by dividing by the mean for a pons and cerebellum reference region (Landau et al., 2011). The FDG-PET meta-ROI was included as a covariate so associations with CBF could be considered independent from this widely used biomarker of cerebral metabolism and AD risk.

\section{Intrusion errors}

The Rey Auditory Verbal Learning Test (AVLT) is a 15-item word-list learning and memory test that includes 5 learning trials, an interference trial with different words, a short-delay 
free recall trial, a 30-min delay free recall trial, and a delayed recognition. The intrusion errors score is the total number of extra-list intrusion errors produced across all learning and delay free recall trials. Prior work has shown that AVLT intrusion errors predict progression from $\mathrm{CU}$ to $\mathrm{MCI}$ and dementia, after adjusting for neuropsychological total scores (Thomas et al., 2018). Because intrusion errors were nonnormally distributed in this sample, a Blom-transformed intrusion errors variable was used in all analyses (Blom, 1958). However, the raw intrusion errors variable was also examined in analyses and results were qualitatively and statistically similar.

\section{Fasting blood glucose}

FBG values were measured as part of the FDG-PET protocol. Participants had blood drawn following a fast of at least $4 \mathrm{~h}$ (water only). For sensitivity analyses, T2DM status was defined based on any of the following: a baseline FBG level of $>125 \mathrm{mg} / \mathrm{dL}$ (American Diabetes Association, 2020), self-report of a T2DM diagnosis, or use of glucose-lowering agents (Li et al., 2016; Thomas, et al., 2020a, b). There were no participants with Type 1 diabetes.

\section{Covariates}

Covariates were included to determine whether associations of FBG and intrusion errors with regional CBF persisted beyond general vascular and $\mathrm{AD}$ risk. Vascular risk was indexed by pulse pressure (a proxy for arterial stiffness, measured as systolic minus diastolic blood pressure; Nation et al., 2013) and body mass index (BMI). APOE $\varepsilon 4$ carrier status was determined by the presence of at least one $\varepsilon 4$ allele. A subset of participants had AD markers from cerebrospinal fluid (CSF) data $(n=97)$. AD CSF markers were processed using Elecsys ${ }^{\circledR}$ immunoassays; biomarker positivity was defined using the previously determined ADNIoptimized phosphorylated tau/ $\beta$-amyloid ( $\mathrm{p}$-tau/A $\beta$ ) ratio cut-score of $>0.0251 \mathrm{pg} / \mathrm{ml}$ (Hansson et al., 2018).

\section{Statistical analyses}

Hierarchical linear regressions were run for each a priori CBF ROI: MTL, IPL, precuneus, mOFC, and pericalcarine (control region). Block 1 included the main effects of FBG and intrusion errors as well as all relevant covariates: age, sex, education, APOE $\varepsilon 4$ status, BMI, pulse pressure, FDG-PET, and reference CBF region (precentral gyrus). Block 2 included the FBG $\mathrm{x}$ intrusion errors interaction to determine whether intrusion errors moderated the relationship between FBG and regional CBF. Sensitivity analyses were run to determine if 1) the results changed when 1) the 14 participants who meet criteria for T2DM were excluded and 2) also adjusting for CSF $\mathrm{p}$-tau/A $\beta$ ratio positivity. Next, follow-up linear regressions assessing regional gray matter volume (MTL) or cortical thickness (IPL, precuneus, mOFC, and pericalcarine), instead of CBF, in the same a priori ROIs were conducted to determine whether the pattern of structural findings are consistent with the CBF findings. The models were identical to the hierarchical models described above, but did not adjust for precentral gyrus CBF. Intracranial volume was included as a covariate in the MTL volume analyses. Potential multicollinearity of the independent variables was assessed for all models and all variance inflation factor (VIF) values were less than 2.

\section{Results}

\section{Effects of blood glucose and intrusion errors on CBF}

Table 2 shows all parameters for the hierarchical linear regressions for each CBF ROI. Briefly, in Block 1, the main effect of FBG was significant such that higher FBG was associated with higher CBF in the precuneus $(\beta=0.134$, $95 \% \mathrm{CI}=0.007$ to $0.261, p=0.039)$, IPL ( $\beta=0.173,95 \%$ $\mathrm{CI}=0.072$ to $0.276, p=0.001)$, and $\mathrm{mOFC}(\beta=0.182,95 \%$ $\mathrm{CI}=0.047$ to $0.320, p=0.009)$ regions. There were no significant main effects of intrusion errors on CBF across ROIs. In Block 2, when the FBG $\mathrm{x}$ intrusion errors interaction was added to the model, the interaction effect was significant such that intrusion errors moderated the relationship between FBG and MTL and precuneus CBF. Specifically, as shown in Fig. 1, having higher FBG levels and more intrusion errors was associated with reduced CBF in the MTL ( $\beta=-0.186,95 \% \mathrm{CI}=-0.334$ to $-0.040, p=0.013)$ and precuneus $(\beta=-0.146,95 \% \mathrm{CI}=-0.273$ to $-0.022, p=0.022)$. The significant results persisted when the false discovery rate was controlled at 0.05 . When hemispheric differences were examined, only the FBG $x$ intrusion errors interaction effect differed for the left and right MTL, such that there was a stronger moderating effect of intrusion errors on FBG for the left MTL CBF $(\beta=-0.188,95 \% \mathrm{CI}=-0.327$ to -0.049 , $p=0.009)$ relative to the right MTL CBF $(\beta=-0.136,95 \%$ $\mathrm{CI}=-0.293$ to $0.020, p=0.086$ ).

\section{CBF sensitivity analyses}

Analyses were re-run after excluding participants with T2DM $(n=14)$. There were no changes to the pattern of FBG $x$ intrusion error interaction results (See Supplemental Table 1). The main effects of FBG on IPL and mOFC $\mathrm{CBF}$ also remained significant, however, the main effect of FBG on precuneus CBF was attenuated $(\beta=0.080,95 \%$ $\mathrm{CI}=-0.071$ to $0.266, p=0.253$ ). 
Table 2 Effects of hierarchical linear regression models examining associations with a priori CBF regions of interest

\begin{tabular}{|c|c|c|c|c|c|c|c|c|c|c|c|c|c|c|c|}
\hline & \multicolumn{3}{|l|}{ MTL } & \multicolumn{3}{|c|}{ Precuneus } & \multicolumn{3}{|c|}{ Inferior Parietal } & \multicolumn{3}{|c|}{$\begin{array}{l}\text { Medial Orbital } \\
\text { Frontal }\end{array}$} & \multicolumn{3}{|c|}{$\begin{array}{l}\text { Pericalcarine (con- } \\
\text { trol) }\end{array}$} \\
\hline & $\beta$ & s.e. & $\mathrm{p}$ & $\beta$ & s.e. & $\mathrm{p}$ & $\beta$ & s.e. & $\mathrm{p}$ & $\beta$ & s.e. & $\mathrm{p}$ & $\beta$ & s.e. & $\mathrm{p}$ \\
\hline \multicolumn{16}{|l|}{ Block 1} \\
\hline Age & .006 & .078 & .937 & -.037 & .066 & .578 & .188 & .053 & .001 & .015 & .071 & .830 & -.084 & .078 & .284 \\
\hline Education & .031 & .079 & .687 & -.042 & .067 & .525 & .091 & .054 & .087 & -.030 & .072 & .671 & .009 & .079 & .914 \\
\hline Female & .022 & .079 & .775 & .090 & .067 & .178 & .223 & .053 & $<.001$ & -.083 & .071 & .247 & .170 & .079 & .035 \\
\hline APOE $\varepsilon 4$ carrier & -.171 & .078 & .030 & -.091 & .066 & .173 & -.036 & .053 & .504 & -.093 & .071 & .193 & -.076 & .079 & .340 \\
\hline BMI & .045 & .075 & .549 & -.046 & .064 & .474 & -.013 & .051 & .803 & -.074 & .068 & .280 & -.003 & .075 & .963 \\
\hline Pulse pressure & -.026 & .079 & .739 & .040 & .067 & .555 & -.112 & .054 & .040 & -.114 & .072 & .118 & .097 & .078 & .224 \\
\hline FDG-PET & -.007 & .079 & .929 & .093 & .067 & .163 & .108 & .054 & .044 & .056 & .072 & .428 & .043 & .080 & .584 \\
\hline Precentral gyrus CBF & .622 & .075 & $<.001$ & .747 & .064 & $<.001$ & .758 & .051 & $<.001$ & .730 & .069 & $<.001$ & .619 & .075 & $<.001$ \\
\hline FBG & .131 & .076 & .084 & .134 & .064 & .039 & .173 & .051 & .001 & .182 & .069 & .009 & .081 & .076 & .285 \\
\hline Intrusion errors & .003 & .076 & .965 & .037 & .064 & .560 & .005 & .052 & .930 & -.073 & .069 & .288 & .044 & .075 & .565 \\
\hline \multicolumn{16}{|l|}{ Block 2} \\
\hline Age & .011 & .076 & .887 & -.033 & .065 & 609 & .190 & .053 & $<.001$ & .016 & .071 & .819 & -.082 & .077 & .293 \\
\hline Education & .036 & .077 & .634 & -.038 & .066 & .554 & .093 & .053 & .078 & -.029 & .072 & .683 & .011 & .079 & .891 \\
\hline Female & -.012 & .078 & .876 & .063 & .066 & .344 & .208 & .054 & $<.001$ & -.091 & .073 & .213 & .146 & .080 & .071 \\
\hline $\mathrm{APOE} \varepsilon 4$ carrier & -.165 & .076 & .033 & -.086 & .065 & .189 & -.033 & .053 & .537 & -.091 & .071 & .202 & -.073 & .079 & .356 \\
\hline BMI & .034 & .073 & .643 & -.054 & .062 & .385 & -.018 & .051 & .730 & -.077 & .069 & .266 & -.012 & .074 & .879 \\
\hline Pulse pressure & -.040 & .077 & .607 & .029 & .066 & .660 & -.118 & .054 & .030 & -.117 & .072 & .110 & .087 & .078 & .268 \\
\hline FDG-PET & .022 & .078 & .778 & .115 & .066 & .080 & .120 & .054 & .026 & .063 & .073 & .383 & .062 & .080 & .429 \\
\hline Precentral gyrus CBF & .604 & .074 & $<.001$ & .732 & .063 & $<.001$ & .750 & .051 & $<.001$ & .726 & .069 & $<.001$ & .608 & .075 & $<.001$ \\
\hline FBG & .134 & .074 & .072 & .135 & .063 & .033 & .174 & .051 & .001 & .183 & .069 & .009 & .083 & .075 & .269 \\
\hline Intrusion errors & .007 & .074 & .929 & .040 & .063 & .526 & .006 & .051 & .908 & -.072 & .069 & .294 & .046 & .074 & .541 \\
\hline FBG $x$ Intrusion errors & -.186 & .074 & .013 & -.146 & .063 & .022 & -.081 & .051 & .118 & -.043 & .069 & .537 & -.123 & .075 & .104 \\
\hline
\end{tabular}

Bold p-values are significant $p<.05$. Block 1 includes all covariates with FBG and intrusion errors as the primary independent variables; Block 2 added the FBG $\mathrm{x}$ intrusion errors interaction to the model. $C B F=$ cerebral blood flow; $M T L=$ medial temporal lobe; $A P O E=$ apolipoprotein $\mathrm{E}$; $B M I=$ body mass index; $F D G-P E T=[18 \mathrm{~F}]$ fluorodeoxyglucose positron emission tomography; $F B G=$ fasting blood glucose

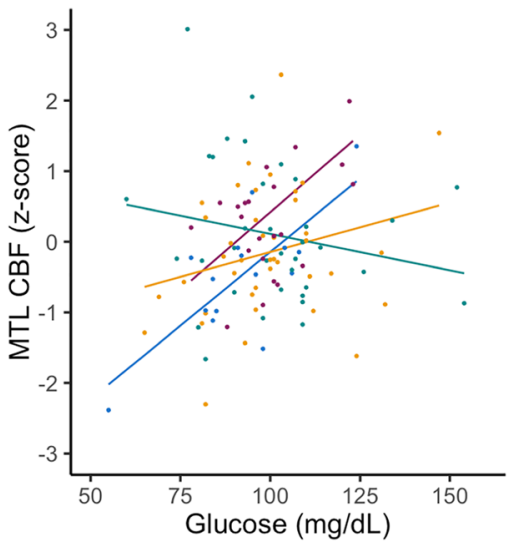

Fig. 1 Intrusions errors moderate the associations between blood glucose and MTL and precuneus CBF. Intrusion errors were a continuous variable in the models, but intrusions were split into quartiles for visual depiction of the FBG $\mathrm{x}$ intrusion errors interaction: 1 st quartile $=0$ Intrusions; 2 nd quartile $=1$ intrusion; 3rd quar-

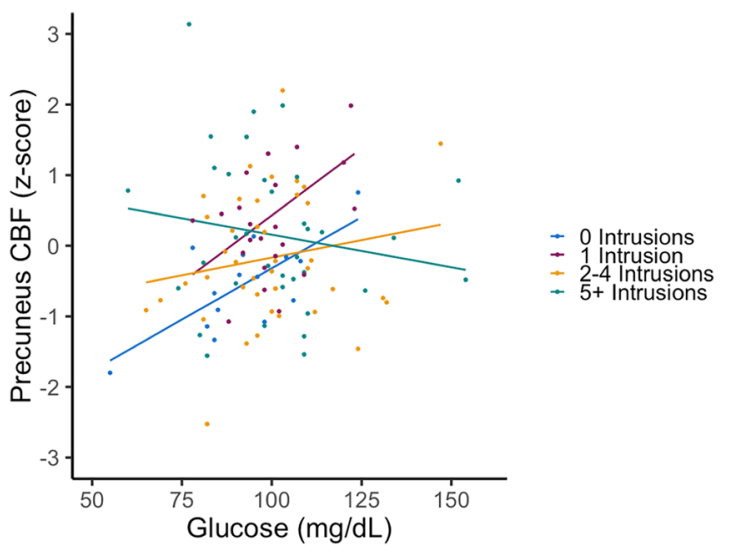

tile $=2-4$ intrusions; 4 th quartile $=5+$ intrusions. Model predicted $\mathrm{CBF}$ is shown in z-score metric and includes adjustment for age, sex, education, APOE $\varepsilon 4$ carrier status, BMI, pulse pressure, FDG-PET, and reference $\mathrm{CBF}$ region (precentral gyrus). $\mathrm{MTL}=$ medial temporal lobe; $\mathrm{CBF}=$ cerebral blood flow; $\mathrm{FBG}=$ fasting blood glucose 
Next, the original analyses were run again after adding in the CSF $\mathrm{p}$-tau/A $\beta$ positivity variable $(n=97$; See Supplemental Table 2). This additional covariate did not change the pattern of results for the FBG $\mathrm{x}$ intrusion errors interaction across any of the CBF ROIs, except the effect on the precuneus was slightly attenuated $(\beta=-0.130,95 \% \mathrm{CI}=-0.272$ to $0.008, p=0.064)$. For MTL, the main effect of FBG was significant $(\beta=0.179,95 \% \mathrm{CI}=0.023$ to $0.325, p=0.025)$ with the addition of the CSF p-tau/A $\beta$ positivity variable such that higher FBG was associated with higher MTL CBF. The pattern of results for any other main effects of FBG or intrusion errors did not change.

\section{Gray matter secondary analyses}

Linear regressions with the same a priori ROIs as the CBF analyses were conducted with regional brain volume/cortical thickness to determine whether the pattern of structural findings is consistent with the CBF findings. In Block 1, there were neither main effects of FBG nor intrusion errors for any of the 5 ROIs. In Block 2, when the FBG $x$ intrusion errors interaction was added to the model, the interaction effect was also not significant across any of the gray matter ROIs (all $p s>0.05$; See Supplemental Table 3).

\section{Discussion}

The current study found that higher FBG was associated with higher CBF in the precuneus, IPL, and mOFC (and MTL only when adjusting for CSF $\mathrm{p}$-tau/A $\beta$ positivity). While there was no main effect of intrusion errors on regional $\mathrm{CBF}$, intrusion errors moderated the relationship between FBG and regional CBF such that higher FBG and more intrusion errors were associated with reduced $\mathrm{CBF}$ in the MTL and precuneus. These interaction effects were largely similar when CSF AD biomarker status was added to the model and persisted when participants with T2DM were excluded.

The main effects of FBG are consistent with prior work that has found higher regional CBF, particularly in the MTL, in participants at risk for progression to AD (e.g., APOE $\varepsilon 4$ carriers, those with subtle cognitive decline), but who are not yet considered cognitively impaired (Bangen et al., 2012; Fleisher et al., 2009; Thomas et al., 2021). These findings may reflect early neurovascular dysregulation, whereby higher $\mathrm{CBF}$ is needed to maintain $\mathrm{CU}$ performance in individuals with higher FBG levels. Conversely, prior work has demonstrated that participants with T2DM show reduced CBF relative to participants without T2DM (Bangen et al., 2018). The reason for these discrepant results may be related to the nature of the samples. For example, ADNI was selected to be a very healthy sample given that only individuals with a modified Hachinski index of $<5$ were included at baseline. The sample in Bangen et al. (2018) was recruited from the community and was also quite healthy. However, all of those participants with T2DM also had hypertension and the sample included both $\mathrm{CU}$ and $\mathrm{MCI}$ participants rather than only CU. Thus, higher FBG levels (consistent with the T2DM diagnosis) plus other risk factors such as MCI or hypertension may be related to hypo- rather than hyperperfusion. Within the current study, all participants were CU, less than half of participants $(40.7 \%)$ had elevated systolic $(>140 \mathrm{mmHg})$ or diastolic $(>90 \mathrm{mmHg})$ blood pressure, and there were only 7 participants with both T2DM and high blood pressure.

The current results found that intrusions errors, a known risk factor for progression to $\mathrm{MCI} / \mathrm{dementia}$ in ADNI (Thomas et al., 2018), moderated the relationship between FBG and regional CBF in the MTL and precuneus (higher FBG level plus greater intrusion errors was associated with lower $\mathrm{CBF}$ ). This finding suggests that while higher regional $\mathrm{CBF}$ is needed to maintain unimpaired performance in people with higher FBG alone, the combination of higher FBG and high intrusion errors may indicate a failure of early compensatory mechanisms that then result in a decrease in neural activity in $\mathrm{AD}$ vulnerable regions (Wierenga et al., 2014). This hypoperfusion, in turn, is then consistent with the known reduced CBF in individuals in the MCI and dementia stages of AD (Bangen et al., 2012; Mattsson et al., 2014) as well as consistent with Zlokovic's two-hit hypothesis of AD pathogenesis (Zlokovic, 2011). Zlokovic proposed that vascular factors such as T2DM ("hit one") result in blood-brain barrier dysfunction and a reduction in CBF that then initiate a cascade of events leading to amyloid accumulation ("hit two"); amyloid and/or hypoperfusion can both induce hyperphosphorylation of tau, leading to neurofibrillary tangle formation and ultimately dementia.

Importantly, the current pattern of results was fairly similar when $\mathrm{p}$-tau/A $\beta$ positivity was adjusted for in the models, suggesting that the observed relationships are likely not dependent on AD pathology. The pattern of results also persisted after excluding participants with T2DM, indicating that even pre-diabetes levels of FBG may negatively impact $\mathrm{CBF}$, especially in the context of an additional AD risk factor (i.e., intrusion errors). These findings are consistent with evidence that pre-diabetes can accelerate cognitive decline (Marseglia et al., 2019) and be associated with greater A $\beta$ on PET (Luchsinger et al., 2020) and more severe MTL neurofibrillary tangle pathology among those at genetic risk for AD (Bangen et al., 2016). Finally, the absence of any main effect of FBG or interaction of FBG and intrusion errors on regional gray matter volumes suggests that these regional CBF patterns may be altered earlier and precede the ultimate downstream structural changes of gray matter degeneration that likely would not be observed until significant tau 
accumulation, supporting $\mathrm{CBF} /$ neurovascular dysfunction as an early biomarker of risk for decline.

A strength of the study is that we were able to characterize and adjust for vascular and metabolic markers that are likely associated with FBG levels and could impact CBF, which allowed us to better understand the unique relationship between FBG, intrusion errors, and regional CBF. The current study was limited by the cross-sectional design, which precludes conclusions about causation and temporal sequence. Importantly, our study is also limited in its generalizability beyond this mostly white, highly educated, and very healthy ADNI sample. It is likely that results may change in populations with higher rates of vascular diseases. However, it is noteworthy that we were able to detect associations with CBF despite ADNI participants being such a selected sample. Notwithstanding the current limitations, if replicated, these results may have important research and clinical implications. Since FBG is a potentially modifiable risk factor for cognitive decline (Livingston et al., 2020), there is need for increased monitoring and potential for optimization of interventions to ensure FBG is within a healthy range, even in individuals without T2DM. In future studies, ASL MRI may be a useful tool for measuring the underlying mechanism of FBG intervention and understanding why change in FBG may be related to change in cognition.

Taken together, our results suggest that regional CBF may be a useful biomarker of early neurovascular changes related to FBG levels, but that these associations may vary according to other risk factors such as subtle cognitive changes. Intrusions errors may be a useful behavioral measure of very early cognitive inefficiency in episodic memory in older adults who are still performing within the normal range on neuropsychological measures, and seem to be particularly meaningful in individuals with higher FBG levels.

Supplementary Information The online version contains supplementary material available at https://doi.org/10.1007/s11682-021-00495-8.

Author contributions Author contributions included conception and study design (KRT, MWB, KJB), data collection or acquisition (KRT, AJW, IHC, ECE, MWB, KJB), statistical analysis (KRT, AJW, KJB), interpretation of results (KRT, CEW, KJB), drafting the manuscript work or revising it critically for important intellectual content (KRT, AJW, IHC, ECE, CEW, MWB, KJB) and approval of final version to be published and agreement to be accountable for the integrity and accuracy of all aspects of the work (All authors).

Funding This work was supported by the U.S. Department of Veterans Affairs Clinical Sciences Research and Development Service (Career Development Award-2 1IK2CX001865 to KRT and 1IK2CX001415 to ECE; Merit Award 1I01CX001842 to KJB and 5I01CX000565 to CEW), NIH grants (R03 AG070435 to KRT, R01 AG063782 to KJB; R01 AG049810 and R01 AG054049 to MWB), and the Alzheimer's Association (AARF-17-528918 to KRT, AARG-18-566254 to KJB, AARG-17-500358 to ECE).

Data collection and sharing for this project was funded by the Alzheimer's Disease Neuroimaging Initiative (ADNI) (National Institutes of Health Grant U01 AG024904) and DOD ADNI (Department of Defense award number W81XWH-12-2-0012). ADNI is funded by the National Institute on Aging, the National Institute of Biomedical Imaging and Bioengineering, and through generous contributions from the following: AbbVie, Alzheimer's Association; Alzheimer's Drug Discovery Foundation; Araclon Biotech; BioClinica, Inc.; Biogen; Bristol-Myers Squibb Company; CereSpir, Inc.; Cogstate; Eisai Inc.; Elan Pharmaceuticals, Inc.; Eli Lilly and Company; EuroImmun; F. Hoffmann-La Roche Ltd and its affiliated company Genentech, Inc.; Fujirebio; GE Healthcare; IXICO Ltd.; Janssen Alzheimer Immunotherapy Research \& Development, LLC.; Johnson \& Johnson Pharmaceutical Research \& Development LLC.; Lumosity; Lundbeck; Merck \& Co., Inc.; Meso Scale Diagnostics, LLC.; NeuroRx Research; Neurotrack Technologies; Novartis Pharmaceuticals Corporation; Pfizer Inc.; Piramal Imaging; Servier; Takeda Pharmaceutical Company; and Transition Therapeutics. The Canadian Institutes of Health Research is providing funds to support ADNI clinical sites in Canada. Private sector contributions are facilitated by the Foundation for the National Institutes of Health (www.fnih.org). The grantee organization is the Northern California Institute for Research and Education, and the study is coordinated by the Alzheimer's Therapeutic Research Institute at the University of Southern California. ADNI data are disseminated by the Laboratory for Neuro Imaging at the University of Southern California.

ADNI was approved by the institutional review boards at each of the participating institutions, and written informed consent was obtained from all participants or authorized representatives at each site.

\section{Declarations}

Conflict of interest/disclosures Dr. Bondi receives royalties from Oxford University Press and serves as a consultant for Eisai, Novartis, and Roche Pharmaceutical. Other authors report no disclosures.

Open Access This article is licensed under a Creative Commons Attribution 4.0 International License, which permits use, sharing, adaptation, distribution and reproduction in any medium or format, as long as you give appropriate credit to the original author(s) and the source, provide a link to the Creative Commons licence, and indicate if changes were made. The images or other third party material in this article are included in the article's Creative Commons licence, unless indicated otherwise in a credit line to the material. If material is not included in the article's Creative Commons licence and your intended use is not permitted by statutory regulation or exceeds the permitted use, you will need to obtain permission directly from the copyright holder. To view a copy of this licence, visit http://creativecommons.org/licenses/by/4.0/.

\section{References}

Abulafia, C., Loewenstein, D., Curiel-Cid, R., Duarte-Abritta, B., Sánchez, S. M., Vigo, D. E., Castro, M. N., Drucaroff, L. J., Vázquez, S., Sevlever, G., Nemeroff, C. B., Guinjoan, S. M., \& Villarreal, M. F. (2018). Brain Structural and Amyloid Correlates of Recovery From Semantic Interference in Cognitively Normal Individuals With or Without Family History of Late-Onset Alzheimer's Disease. The Journal of Neuropsychiatry and Clinical Neurosciences, 31(1), 25-36. https://doi.org/10.1176/appi.neuro psych. 17120355

American Diabetes Association. (2020). 2. Classification and Diagnosis of Diabetes: Standards of Medical Care in Diabetes-2020. Diabetes Care, 43(Supplement 1), S14-S31. https://doi.org/10. 2337/dc20-S002 
Bangen, K. J., Clark, A. L., Edmonds, E. C., Evangelista, N. D., Werhane, M. L., Thomas, K. R., Locano, L. E., Tran, M., Zlatar, Z. Z., Nation, D. A., Bondi, M. W., \& Delano-Wood, L. (2017). Cerebral Blood Flow and Amyloid- $\beta$ Interact to Affect Memory Performance in Cognitively Normal Older Adults. Frontiers in Aging Neuroscience, 9. https://doi.org/10.3389/fnagi.2017.00181

Bangen, K. J., Himali, J. J., Beiser, A. S., Nation, D. A., Libon, D. J., Fox, C. S., Seshadri, S., Wolf, P. A., McKee, A. C., Au, R., \& Delano-Wood, L. (2016). Interaction Between Midlife Blood Glucose and APOE Genotype Predicts Later Alzheimer's Disease Pathology. Journal of Alzheimer's Disease, 53(4), 1553-1562. https://doi.org/10.3233/JAD-160163

Bangen, K. J., Nation, D. A., Delano-Wood, L., Weissberger, G. H., Hansen, L. A., Galasko, D. R., Salmon, D. P., \& Bondi, M. W. (2015). Aggregate effects of vascular risk factors on cerebrovascular changes in autopsy-confirmed Alzheimer's disease. Alzheimer's \& Dementia, 11(4), 394-403.e1. https://doi.org/10.1016/j. jalz.2013.12.025

Bangen, K. J., Restom, K., Liu, T. T., Wierenga, C. E., Jak, A. J., Salmon, D. P., \& Bondi, M. W. (2012). Assessment of Alzheimer's Disease Risk with Functional Magnetic Resonance Imaging: An Arterial Spin Labeling Study. Journal of Alzheimer's Disease, 31(s3), S59-S74. https://doi.org/10.3233/JAD-2012-120292

Bangen, K. J., Werhane, M. L., Weigand, A. J., Edmonds, E. C., Delano-Wood, L., Thomas, K. R., Nation, D. A., Evangelista, N. D., Clark, A. L., Liu, T. T., \& Bondi, M. W. (2018). Reduced Regional Cerebral Blood Flow Relates to Poorer Cognition in Older Adults With Type 2 Diabetes. Frontiers in Aging Neuroscience, 10. https://doi.org/10.3389/fnagi.2018.00270

Blom, G. (1958). Statistical estimates and transformed beta-variables. Wiley.

Bondi, M. W., Edmonds, E. C., Jak, A. J., Clark, L. R., Delano-Wood, L., McDonald, C. R., Nation, D. A., Libon, D. J., Au, R., Galasko, D., Salmon, D. P., \& Initiative, for the A. D. N. . (2014). Neuropsychological Criteria for Mild Cognitive Impairment Improves Diagnostic Precision, Biomarker Associations, and Progression Rates. Journal of Alzheimer's Disease, 42(1), 275-289. https:// doi.org/10.3233/JAD-140276

Bondi, M. W., Salmon, D. P., Galasko, D., Thomas, R. G., \& Thal, L. J. (1999). Neuropsychological function and apolipoprotein $\mathrm{E}$ genotype in the preclinical detection of Alzheimer's disease. Psychology and Aging, 14(2), 295.

Dickerson, B. C., Stoub, T. R., Shah, R. C., Sperling, R. A., Killiany, R. J., Albert, M. S., Hyman, B. T., Blacker, D., \& deToledoMorrell, L. (2011). Alzheimer-signature MRI biomarker predicts AD dementia in cognitively normal adults. Neurology, 76(16), 1395-1402. https://doi.org/10.1212/WNL.0b013e3182166e96

Fleisher, A. S., Podraza, K. M., Bangen, K. J., Taylor, C., Sherzai, A., Sidhar, K., Liu, T. T., Dale, A. M., \& Buxton, R. B. (2009). Cerebral perfusion and oxygenation differences in Alzheimer's disease risk. Neurobiology of Aging, 30(11), 1737-1748. https:// doi.org/10.1016/j.neurobiolaging.2008.01.012

Hansson, O., Seibyl, J., Stomrud, E., Zetterberg, H., Trojanowski, J. Q., Bittner, T., Lifke, V., Corradini, V., Eichenlaub, U., Batrla, R., Buck, K., Zink, K., Rabe, C., Blennow, K., \& Shaw, L. M. (2018). CSF biomarkers of Alzheimer's disease concord with amyloid- $\beta$ PET and predict clinical progression: A study of fully automated immunoassays in BioFINDER and ADNI cohorts. Alzheimer's \& Dementia, 14(11), 1470-1481. https://doi.org/10.1016/j.jalz. 2018.01.010

Jagust, W. J., Bandy, D., Chen, K., Foster, N. L., Landau, S. M., Mathis, C. A., Price, J. C., Reiman, E. M., Skovronsky, D., \& Koeppe, R. A. (2010). The Alzheimer's Disease Neuroimaging Initiative positron emission tomography core. Alzheimer's \& Dementia, 6(3), 221-229. https://doi.org/10.1016/j.jalz.2010.03.003
Jak, A. J., Bondi, M. W., Delano-Wood, L., Wierenga, C., CoreyBloom, J., Salmon, D. P., \& Delis, D. C. (2009). Quantification of Five Neuropsychological Approaches to Defining Mild Cognitive Impairment. The American Journal of Geriatric Psychiatry, 17(5), 368-375. https://doi.org/10.1097/JGP.0b013e31819431d5

Kapasi, A., DeCarli, C., \& Schneider, J. A. (2017). Impact of multiple pathologies on the threshold for clinically overt dementia. Acta Neuropathologica, 134(2), 171-186. https://doi.org/10.1007/ s00401-017-1717-7

Landau, S. M., Harvey, D., Madison, C. M., Koeppe, R. A., Reiman, E. M., Foster, N. L., Weiner, M. W., \& Jagust, W. J. (2011). Associations between cognitive, functional, and FDG-PET measures of decline in AD and MCI. Neurobiology of Aging, 32(7), 12071218. https://doi.org/10.1016/j.neurobiolaging.2009.07.002

Lee, S., Viqar, F., Zimmerman, M. E., Narkhede, A., Tosto, G., Benzinger, T. L. S., Marcus, D. S., Fagan, A. M., Goate, A., Fox, N. C., Cairns, N. J., Holtzman, D. M., Buckles, V., Ghetti, B., McDade, E., Martins, R. N., Saykin, A. J., Masters, C. L., Ringman, J. M., ... Brickman, A. M. (2016). White matter hyperintensities are a core feature of Alzheimer's disease: Evidence from the dominantly inherited Alzheimer network. Annals of Neurology, 79(6), 929-939. https://doi.org/10.1002/ana.24647

Li, W., Risacher, S. L., Huang, E., Saykin, A. J., \& Initiative, A. D. N. (2016). Type 2 diabetes mellitus is associated with brain atrophy and hypometabolism in the ADNI cohort. Neurology, 87(6), 595-600.

Livingston, G., Huntley, J., Sommerlad, A., Ames, D., Ballard, C., Banerjee, S., Brayne, C., Burns, A., Cohen-Mansfield, J., Cooper, C., Costafreda, S. G., Dias, A., Fox, N., Gitlin, L. N., Howard, R., Kales, H. C., Kivimäki, M., Larson, E. B., Ogunniyi, A., ... Mukadam, N. (2020). Dementia prevention, intervention, and care: 2020 report of the Lancet Commission. The Lancet, 396(10248), 413-446. https://doi.org/10.1016/S0140-6736(20)30367-6

Loewenstein, D. A., Curiel, R. E., Duara, R., \& Buschke, H. (2018). Novel Cognitive Paradigms for the Detection of Memory Impairment in Preclinical Alzheimer's Disease. Assessment, 25(3), 348-359. https://doi.org/10.1177/1073191117691608

Luchsinger, J. A., Palta, P., Rippon, B., Sherwood, G., Soto, L., Ceballos, F., Laing, K., Igwe, K., He, H., Razlighi, Q., Teresi, J., Moreno, H., \& Brickman, A. M. (2020). Pre-Diabetes, but not Type 2 Diabetes, Is\&nbsp;Related to Brain Amyloid in Late Middle-Age. Journal of Alzheimer's Disease, 75(4), 1241-1252. https://doi.org/10.3233/JAD-200232

Luchsinger, J. A., Reitz, C., Patel, B., Tang, M.-X., Manly, J. J., \& Mayeux, R. (2007). Relation of Diabetes to Mild Cognitive Impairment. Archives of Neurology, 64(4), 570-575. https://doi. org/10.1001/archneur.64.4.570

Luh, W.-M., Wong, E. C., Bandettini, P. A., \& Hyde, J. S. (1999). QUIPSS II with thin-slice TI1 periodic saturation: A method for improving accuracy of quantitative perfusion imaging using pulsed arterial spin labeling. Magnetic Resonance in Medicine, 41(6), 1246-1254.

Marseglia, A., Fratiglioni, L., Kalpouzos, G., Wang, R., Bäckman, L., $\& \mathrm{Xu}, \mathrm{W}$. (2019). Prediabetes and diabetes accelerate cognitive decline and predict microvascular lesions: A population-based cohort study. Alzheimer's \& Dementia, 15(1), 25-33. https://doi. org/10.1016/j.jalz.2018.06.3060

Mattsson, N., Tosun, D., Insel, P. S., Simonson, A., Jack, C. R., Beckett, L. A., Donohue, M., Jagust, W., Schuff, N., \& Weiner, M. W. (2014). Association of brain amyloid- $\beta$ with cerebral perfusion and structure in Alzheimer's disease and mild cognitive impairment. Brain, 137(5), 1550-1561. https://doi.org/10.1093/brain/ awu043

McKhann, G., Drachman, D., Folstein, M., Katzman, R., Price, D., \& Stadlan, E. M. (1984). Clinical diagnosis of Alzheimer's disease: Report of the NINCDS-ADRDA Work Group* under the auspices 
of Department of Health and Human Services Task Force on Alzheimer's Disease. Neurology, 34(7), 939-939.

Nation, D. A., Edland, S. D., Bondi, M. W., Salmon, D. P., DelanoWood, L., Peskind, E. R., Quinn, J. F., \& Galasko, D. R. (2013). Pulse pressure is associated with Alzheimer biomarkers in cognitively normal older adults. Neurology, 81(23), 2024-2027.

Petersen, R. C., Aisen, P. S., Beckett, L. A., Donohue, M. C., Gamst, A. C., Harvey, D. J., Jack, C. R., Jagust, W. J., Shaw, L. M., \& Toga, A. W. (2010). Alzheimer's disease neuroimaging initiative (ADNI): Clinical characterization. Neurology, 74(3), 201-209.

Sanchez, D. L., Thomas, K. R., Edmonds, E. C., Bondi, M. W., Bangen, K. J., \& Initiative, for the A. D. N. . (2020). Regional Hypoperfusion Predicts Decline in Everyday Functioning at Three-Year Follow-Up in Older Adults without Dementia. Journal of Alzheimer's Disease, 77(3), 1291-1304. https://doi.org/10.3233/JAD-200490

Thomas, K. R., Bangen, K. J., Weigand, A. J., Edmonds, E. C., Sundermann, E., Wong, C. G., Eppig, J., Werhane, M. L., Delano-Wood, L., Bondi, M. W., \& Initiative, for the A. D. N. . (2020a). Type 2 Diabetes Interacts With Alzheimer Disease Risk Factors to Predict Functional Decline. Alzheimer Disease \& Associated Disorders, 34(1), 10-17. https://doi.org/10.1097/WAD.0000000000000332

Thomas, K. R., Eppig, J., Edmonds, E. C., Jacobs, D. M., Libon, D. J., $\mathrm{Au}$, R., Salmon, D. P., \& Bondi, M. W. (2018). Word-list intrusion errors predict progression to mild cognitive impairment. Neuropsychology, 32(2), 235-245. https://doi.org/10.1037/neu00 00413
Thomas, K. R., Osuna, J. R., Weigand, A. J., Edmonds, E. C., Clark, A. L., Holmquist, S., Cota, I. H., Wierenga, C. E., Bondi, M. W., \& Bangen, K. J. for the Alzheimer's Disease Neuroimaging Initiative. (2021). Regional hyperperfusion in older adults with objectively-defined subtle cognitive decline. Journal of Cerebral Blood Flow \& Metabolism, 41(5), 1001-1012.

Wierenga, C. E., Hays, C. C., \& Zlatar, Z. Z. (2014). Cerebral Blood Flow Measured by Arterial Spin Labeling MRI as a Preclinical Marker of Alzheimer's Disease. Journal of Alzheimer's Disease, 42(s4), S411-S419. https://doi.org/10.3233/JAD-141467

Yaffe, K., Blackwell, T., Kanaya, A. M., Davidowitz, N., Barrett-Connor, E., \& Krueger, K. (2004). Diabetes, impaired fasting glucose, and development of cognitive impairment in older women. Neurology, 63(4), 658-663.

Yew, B., \& Nation, D. A. (2017). Cerebrovascular resistance: Effects on cognitive decline, cortical atrophy, and progression to dementia. Brain, 140(7), 1987-2001. https://doi.org/10.1093/brain/awx112

Zlokovic, B. V. (2011). Neurovascular pathways to neurodegeneration in Alzheimer's disease and other disorders. Nature Reviews Neuroscience, 12(12), 723-738. https://doi.org/10.1038/nrn3114

Publisher's note Springer Nature remains neutral with regard to jurisdictional claims in published maps and institutional affiliations. 